\title{
Aprendizagem de uma habilidade motora seriada em diferentes estágios de desenvolvimento
}

CDD. 20.ed. 152.3

\author{
Ariane Brito DINIZ* \\ Luciano BASSO ${ }^{* *}$ \\ Debora HASHIGUCHI ${ }^{*}$ \\ Natália Barros BELTRÃO* \\ Elisa Renata Freitas Guerra CORREIA* \\ Dayana da Silva OLIVEIRA* \\ Ilana Santos de OLIVEIRA* \\ Maria Teresa CATTUZZO* \\ *Escola Superior de \\ Educação Física, Uni- \\ versidade de Pernam- \\ cola de Educa- \\ ção Física e Esporte, \\ Universidade de São \\ Paulo.
}

\section{Resumo}

Este estudo investigou a aprendizagem de uma tarefa motora seriada em diferentes estágios de desenvolvimento. Quinze crianças, 14 adultos e 13 idosos praticaram a tarefa de rastrear uma sequência de seis estímulos luminosos durante 10 blocos de tentativas ou até descobrir a sequência, constituindo a fase de estabilização e mais dois blocos de tentativas, referentes as fases de adaptação I e II. 0 desempenho foi mensurado por meio das respostas funcionais e não-funcionais e das sequências funcionais. Os resultados indicaram que os adultos foram superiores aos demais participantes, e idosos apresentaram melhor desempenho que crianças apenas no início da prática, sugerindo que o estágio de desenvolvimento interage com o processo de aprendizagem motora.

UnITERMOS: Aprendizagem; Desempenho psicomotor; Tarefa de rastreamento; Padrão serial.

\section{Introdução}

Estudos sobre a aquisição de habilidades motoras nas subáreas desenvolvimento motor e aprendizagem motora tradicionalmente têm sido diferenciados por fatores como o tempo de observação das mudanças, a importância da prática sistematizada, o efeito da maturação, o enfoque intra ou inter-tarefas. No entanto, o complexo e mal-definido fenômeno da aquisição de habilidades motoras (CHOSHI, 2000) começa a suscitar o alargamento dessas fronteiras que delimitam sua explicação. Com base numa revisão dos estudos sobre aprendizagem, controle e desenvolvimento motor que foram publicados na Research Quarterly for Exercise and Sport, Ulrich e ReEve (2005) propuseram a quebra das tradicionais barreiras que separam e limitam a apreciação dos estudos nesses campos, o que seria o mesmo que dizer que dever-se-ia abordar o estudo da aquisição de habilidades motoras humanas em um esforço mais integrado.

PalAZZIN (2007) tem enfatizado a importância dos estudos de aprendizagem em diferentes estágios de desenvolvimento, uma vez que a maioria é feita com adultos. Além disso, a descrição dos eventos ao longo do processo de desenvolvimento humano é uma importante etapa nas teorias sobre as mudanças do comportamento, pois complementa o poder explanatório de tais teorias, indicando não só o momento da ocorrência dos eventos, mas também sua interação com aqueles que ocorreram antes e depois (Hopkins, Kalveboer \& Geuze, 1993).

Analisando estudos que investigaram o efeito do desenvolvimento na aprendizagem pode-se argumentar que os idosos, em relação aos sujeitos mais jovens, tendem a necessitar de maior tempo de resposta tanto em tarefas simples (Fraser, Li \& Penhune, 2010) quanto complexas (ABreu, 2000; Fraser, Li \& Penhune, 2010; Kemper, Herman \& Lian, 2003; PINHEIRO \& CORRÊA, 2005). A explicação baseia-se nas exigências do processamento de informação (PINHEIRO \& CORREA, 2005) e nas limitações da memória de trabalho (ERVEN \& JANCZURA, 2004). 
Num estudo sobre fatores que afetam a aprendizagem motora de crianças mais jovens e mais velhas, NeWell e KenNedy (1978) concluíram que as mais jovens têm um processamento de informação menos eficiente. De acordo com PaLAzZin (2007), os aspectos cognitivos relativos à formação de modelos internos do movimento (representaçôes), relacionam-se à maturidade do sistema nervoso das crianças e poderiam explicar as desvantagens dos aprendizes mais novos.

Considerando a aprendizagem motora um fenômeno contínuo (TANI, CORRÊA, BENDA \& MANOEL, 2005), crianças e idosos não deveriam ser pensados apenas como processadores de informação melhores ou piores, mas que, ao apresentarem-se como sistemas de diferentes níveis de complexidade, talvez lidem com a informação de forma qualitativamente diferente. Em termos operacionais, parece ser necessário ultrapassar os métodos que avaliam a aprendizagem testando a formação e generalização da estrutura mental. Uma forma seria partir para métodos que imponham desafios ao potencial adaptativo do sistema perceptual-motor, instigando o aprendiz a responder a perturbações. Esse seria um modo válido de avaliar a competência de um sistema aberto e dinâmico que deve ajustar-se continuamente às mais variadas demandas perceptuais e motoras.

\section{Métodos}

\section{Amostra}

Este é um estudo descritivo transversal de comparação entre grupos (SAMPIERI, Collado \& LúCiO, 2006; Thomas \& Nelson, 2002). Participaram deste estudo 42 sujeitos voluntários, recrutados mediante convite verbal, pareados de acordo com o sexo. Os indivíduos foram separados em três grupos de acordo com a faixa etária, 15 crianças $(\hat{\partial}, \mathrm{n}=7 ; 9,89 \pm 0,5$ anos; + , $\mathrm{n}=8 ; 9,82 \pm 0,3), 14$ adultos ( $0, \mathrm{n}=7$; $23,52 \pm 3,1$ anos; $9, \mathrm{n}=7 ; 21,36 \pm 1,5)$ e 13 idosos ( ${ }^{\lambda}, \mathrm{n}=5 ; 70,30 \pm 7,6$ anos; + , $\left.\mathrm{n}=8 ; 70,88 \pm 4,7\right)$. As crianças eram oriundas da Escola Herbert de Souza, Recife - PE, os adultos jovens cursavam a graduação em Educação Física da ESEF/UPE e os idosos eram participantes do Projeto Master Vida da ESEF/UPE.

Adultos, idosos e responsáveis assinaram o termo de consentimento livre e esclarecido (TCLE), aprovado pelo Comitê de Ética da Universidade de Pernambuco (protocolo do CEP-UPE n ${ }^{\circ}$ 87/08; CAAE - 0085.0.097.000-08) e, para o grupo de idosos
Para a elaboração de uma situação que exija organizar aspectos perceptuais-motores o presente estudo utilizou uma tarefa de rastreamento de um padrão luminoso seriado. Neste tipo de tarefa os aprendizes têm que responder a estímulos que aparecem em sequência, e respostas a sequências de estímulos podem ser encontradas em muitas situações cotidianas. O sucesso da tarefa depende da elaboração e execução de uma sequência motora coordenada com a sequência de estímulos luminosos. É possível analisar o desempenho mediante respostas que são qualitativamente diferentes: respostas omissas, erradas, corretas e antecipadas. A prática deve fazer aumentar o percentual de respostas corretas e antecipadas - chamadas de respostas funcionais, pois atendem a meta da tarefa - e uma modificação em algum dos elementos tarefa/ambiente/aprendiz pode provocar (ou não) alteração em tais respostas.

Assim, considerando a aprendizagem como um processo contínuo, pode-se questionar se o estágio do desenvolvimento é capaz de interagir com a formação da estrutura e a competência adaptativa. Desta forma o presente estudo objetivou investigar crianças, adultos e idosos durante a aprendizagem de uma tarefa seriada, comparando o desempenho dos grupos durante a formação da estrutura e suas respostas adaptativas diante de perturbações.

foi controlada a presença da variável interveniente déficit mental. Para o déficit mental dos idosos utilizamos o Mini-Exame do Estado Mental - MEEM, adotando os seguintes escores de escolaridade como ponto de corte: 21 pontos (sem escolarização), 25 pontos (de quatro a oito anos de escolarização) e 28 pontos (acima de nove anos de escolarização) (BRUCKI, Nitrini, Caramelli, Betolucci \& Okamoto, 2003). Nenhum dos participantes tinha experiência anterior com a tarefa a ser aprendida.

\section{Procedimentos}

Foi utilizado o Sistema para Rastreamento de Tarefas Seriais (SRTT), o qual é composto por um hardware, um computador de controle, seis sensores de tempo sensíveis ao toque e seis emissores de estímulos luminosos. Os sensores foram apoiados sobre uma mesa, linearmente alinhados e ficavam distantes $5,0 \mathrm{~cm}$ da borda anterior da mesa, $2,0 \mathrm{~cm} \mathrm{da}$ borda lateral direita e 2,0 cm entre si (FIGURA 1). 


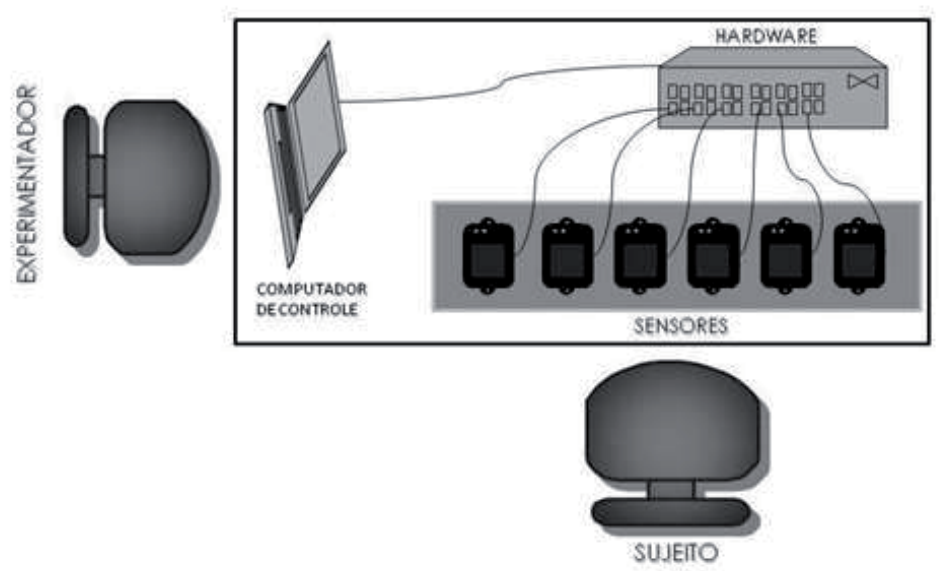

FIGURA 1 - Esquema do dispositivo experimental, ilustrando a disposição dos sensores em série e a posição do sujeito e do experimentador durante a coleta dos dados.

A tarefa consistiu em tocar com o dedo indicador da mão dominante seis sensores de tempo em correspondência aos estímulos luminosos, que se acendiam em uma dada ordem, de forma a descobrir o padrão na ordem de acendimento dos mesmos. A ordem utilizada 3-1-4-6-2-5 já foi usada em estudo anterior (CATTUZZO, 2007), era desafiante, evitava "runs" (2-3-4), "trills" (5-4-5-4) e "repetitions" (1-11) (RestLE, 1970), e a quantidade máxima de prática foi definida em estudo piloto, observando melhora de desempenho sem fadiga ou perda de motivação.

Os participantes sentaram à mesa, de frente para os sensores. Foram informados que seis luzes acenderiam em sequência, que o objetivo era acompanhar o acendimento das luzes tocando os sensores correspondentes, e que o teste terminava quando as luzes parassem de acender. Nessa fase foi usado o intervalo intertentativas (IIE) de $500 \mathrm{~ms}$; os participantes praticavam cinco sequências, totalizando 30 tentativas, e puderam tirar dúvidas após isso. Ao final da familiarização, o experimentador mudava a ordem de acendimento das luzes e após avisar os participantes do início do teste, tinha início a fase de estabilização.

A fase de estabilização compreendia 10 blocos (E1 a E10), cada bloco consistia de 10 sequências com 60 tentativas cada, com o mesmo IIE da familiarização. Ao final de cada bloco o experimentador perguntava ao participante se este era capaz de identificar o padrão seriado dos estímulos luminosos e se podia repeti-lo tocando os sensores (critério declarativo procedimental). Em caso de resposta negativa o indivíduo continuava praticando até alcançar o critério de desempenho ou até a prática do décimo bloco; em caso de resposta positiva o praticante passava para a próxima fase. $\mathrm{O}$ objetivo desta fase era formar uma estrutura mental para a tarefa motora seriada.

Ao final do décimo bloco ou após ter alcançado o critério de estabilização iniciava-se a fase de adaptação I (AdI), cujo objetivo era inserir uma modificação para testar o potencial adaptativo da estrutura mental formada na estabilização. Assim, nesta fase, foi introduzido um intervalo de cinco minutos, antes do início da prática de mais dois blocos compostos de 10 sequências cada, mantendo a ordem de acendimento das luzes e o IIE da fase anterior. Após a AdI, foi dado um intervalo de mais cinco minutos e os sujeitos foram submetidos à fase de adaptação II (AdII), mantendo-se a ordem de acendimento das luzes, e inserindo-se uma nova modificação por meio da diminuição do IIE para $400 \mathrm{~ms}$, em mais dois blocos de 10 sequências cada, ou seja, na AdII o objetivo foi submeter, intencionalmente, a estrutura a uma nova e mais exigente situação. Todos os sujeitos participaram de todas as etapas da tarefa utilizando os mesmos critérios e o QUADRO 1 resume as fases do experimento. 
QUADRO 1 - Fases do experimento e suas características.

\begin{tabular}{|l|c|c|c|c|}
\hline \multicolumn{1}{|c|}{ Fases } & Familiarização & Estabilização & Adaptação I & Adaptação II \\
\hline Blocos & 1 & Até 10 & 2 & 2 \\
\hline Sequências & 5 & 10 & 2 & 2 \\
\hline Tentativas por bloco & 30 & 60 & 60 & $400 \mathrm{~ms}$ \\
\hline IIE & $500 \mathrm{~ms}$ & $500 \mathrm{~ms}$ & $500 \mathrm{~ms}$ & 5 minutos de inter- \\
\hline Perturbação & Não houve & Não houve & $\begin{array}{c}5 \text { minutos de inter- } \\
\text { valo antes da prática }\end{array}$ & $\begin{array}{c}5 \text { valo antes da prática } \\
\text { e diminuição de IIE }\end{array}$ \\
\hline
\end{tabular}

\section{Medidas de desempenho}

As possíveis respostas na tarefa de rastreamento podem ser organizadas hierarquicamente em: omissas, erradas, corretas e antecipadas. A resposta omissa ocorre quando o indivíduo nem consegue esboçar uma resposta ao estímulo; a resposta errada ocorre quando, para um dado estímulo, o indivíduo responde tocando um sensor não correspondente. Ambas podem ser consideradas não funcionais, uma vez que não atendem à meta da tarefa. Respostas corretas (toque no sensor correto durante o intervalo inter-estímulo) ou antecipadas (toque no sensor correto antes do estímulo luminoso acender) são consideradas respostas funcionais, assim como as sequências funcionais (toque correto e/ou antecipado da sequência dos estímulos), pois atendem à meta. Assim, o desempenho foi medido por meio das respostas funcionais, não funcionais e das sequências funcionais. $\mathrm{O}$ valor zero (0) foi atribuído para as respostas não funcionais e um (1) para as respostas funcionais, as quais foram tabuladas por blocos.
Após a verificação da ausência de normalidade da distribuição dos dados (teste de Shapiro-Wilk) e da homogeneidade da variância (teste de Levene) na maioria dos blocos e grupos, foram realizadas análises não paramétricas. Para apresentar o desempenho dos grupos ao longo dos blocos foi utilizada a mediana. Para todas as análises foi adotado nível de significância de 0,05. No início das análises, verificou-se a condição de semelhança inicial dos grupos com relação ao desempenho em respostas funcionais. O pacote estatístico utilizado foi o SPSS 17.0 ("free download").

Inicialmente foi analisado, em cada grupo, o número de sujeitos cuja estabilização foi observada por meio de alcance de critério declarativo-procedimental ou por meio da prática de até 10 blocos de tentativas. A seguir, para analisar o desempenho dos grupos no diferentes momentos (estabilização AdI e AdII) foi utilizado o percentual de respostas funcionais no início e final da estabilização (E2 e E10) e os últimos blocos das fases de adaptação (AdI_2 e AdII_2 respectivamente).

\section{Resultados}

$\mathrm{Na}$ análise da fase de estabilização pode-se observar que 12 crianças, um adulto e 12 idosos realizaram a prática até o último bloco, e que 13 adultos alcançaram o critério antes do quinto bloco de prática, ou seja, $80 \%$ das crianças e $92,3 \%$ dos idosos praticaram até o último bloco enquanto $92,8 \%$ dos adultos alcançaram o critério até o quinto bloco de prática (TABELA 1).

TABELA 1 - Número deindivíduos na fase deestabilização que praticaram atéo alcance do critério de desempenho ou até completar 10 blocos.

\begin{tabular}{lcccccccccc}
\hline & \multicolumn{10}{c}{ Blocos da Fase de Estabilização } \\
& E1 & E2 & E3 & E4 & E5 & E6 & E7 & E8 & E9 & E10 \\
\hline Total de Crianças & 15 & 15 & 14 & 14 & 14 & 13 & 13 & 13 & 12 & 12 \\
Total de Adultos & 14 & 12 & 9 & 6 & 2 & 1 & 1 & 1 & 1 & 1 \\
Total de Idosos & 13 & 13 & 13 & 13 & 13 & 13 & 13 & 13 & 13 & 12 \\
\hline
\end{tabular}


A FIGURA 2 ilustra o desempenho dos sujeitos em respostas funcionais ao longo da fase de estabilização. A observação do desempenho dos grupos no primeiro bloco de prática da fase de estabilização poderia sugerir superioridade inicial do grupo de adultos, no entanto, o teste de Kruskal Wallis não indicou diferenças significativas $\left(\chi^{2}=1,984 ; \mathrm{p}=\right.$ $0,371)$ nas respostas funcionais entre os grupos, no primeiro bloco da prática. É possível notar a superioridade dos adultos no segundo bloco de prática, e no terceiro bloco a mediana passou a ser zero devido ao fato de que cinquenta por cento ou mais dos adultos chegaram à estabilização por alcance de critério declarativo-procedimental nos blocos iniciais.

Pode-se observar que, descritivamente, os idosos têm desempenho superior às crianças durante quase toda a fase de estabilização, mas o teste de Kruskal Wallis não indicou existir diferenças significativas entre crianças e idosos em respostas funcionais.

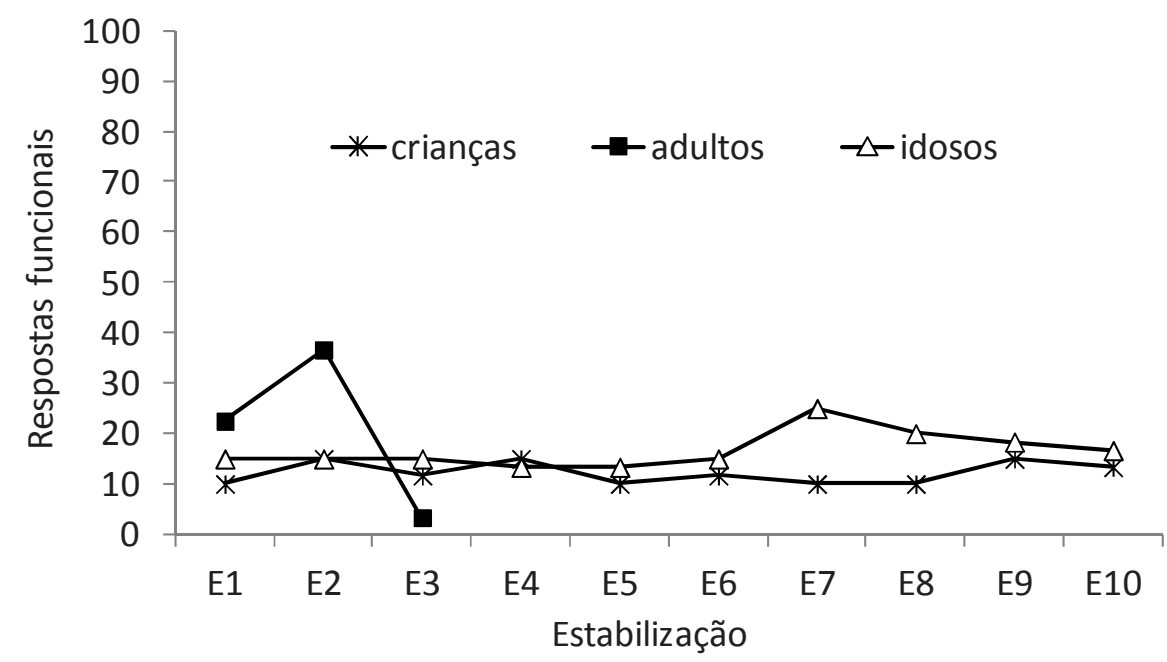

FIGURA 2 - Mediana das respostas funcionais deadultos, crianças eidososnafasedeestabilização (blocosE1atéE10).

As possíveis diferenças entre crianças e idosos passaram a ser exploradas mediante a análise das respostas não-funcionais (omissas e erradas). Descritivamente os idosos mostraram tendência ao menor número das respostas não-funcionais omissas (FIGURA 3); a análise inferencial confirmou esse desempenho dos idosos em respostas omissas apenas no início da prática (bloco E1) $\left(\chi^{2}=5,352\right.$; $\mathrm{p}=0,021)$.

A TABELA 2 ilustra o desempenho dos grupos no início e no final da estabilização e ao responderem às modificaçōes impostas nas fases de adaptação I e II.

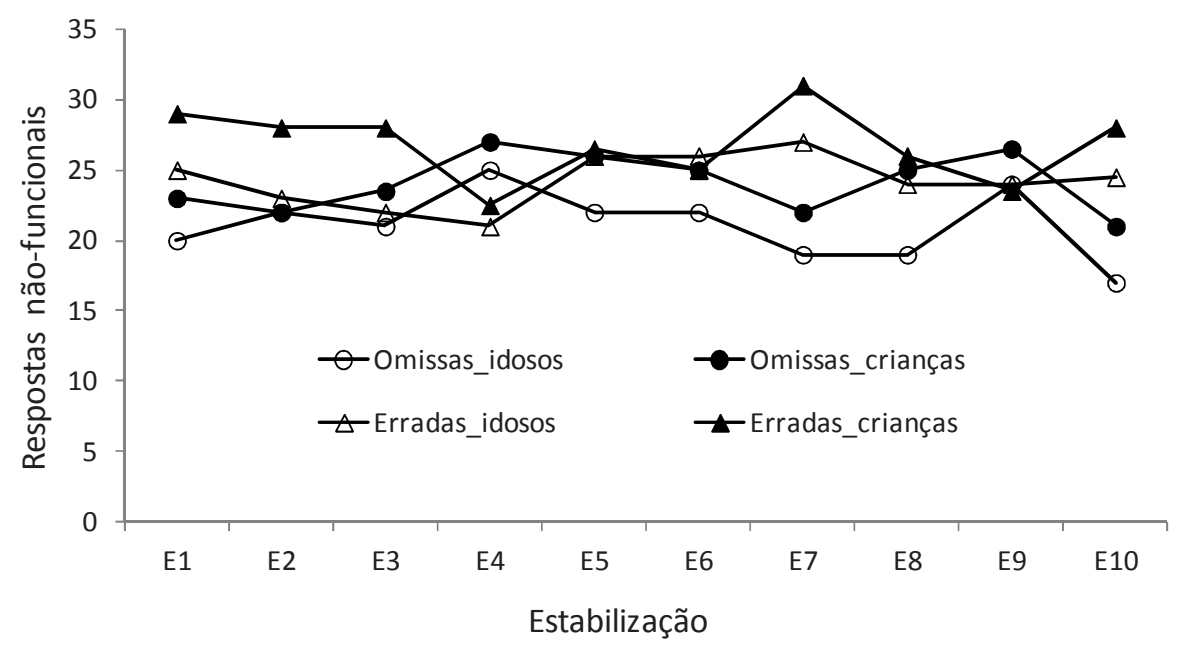

FIGURA 3 - Mediana das respostas funcionais deadultos, crianças eidososnafasedeestabilização (blocosE1atéE10). 
TABELA 2 - Mediana da porcentagem de respostas funcionais de adultos, crianças eidosos no início e no final da fase de estabilização (Blocos E2 eE10) eas respostas às modificações no último bloco da AdI (AdI_2) e no último bloco da AdII (AdII_2)).

\begin{tabular}{lcccc}
\hline \multicolumn{1}{c}{ Grupo/Bloco } & $\begin{array}{c}\text { E02 } \\
\mathbf{( \% )}\end{array}$ & $\begin{array}{c}\text { E10 } \\
\mathbf{( \% )}\end{array}$ & $\begin{array}{c}\text { AdI_2 } \\
\mathbf{( \% )}\end{array}$ & $\begin{array}{c}\text { AdII_2 } \\
\mathbf{( \% )}\end{array}$ \\
\hline Crianças & 15,83 & 13,33 & 18,33 & 11,67 \\
Adultos & 36,67 & $-\ldots--$ & 79,17 & 92,5 \\
Idosos & 15,00 & 16,67 & 23,33 & 13,33 \\
\hline
\end{tabular}

No grupo das crianças o teste de Friedman indicou diferença significativa entre os diferentes momentos do experimento $\left(\chi^{2}=15,561 ; \mathrm{p}=0,001\right)$. O teste de Wilcoxon usado como "post hoc" indicou que houve aumentos significativos de respostas funcionais entre o início da estabilização (E2) e a AdI ( $Z$ $=-2,073, p=0,038)$ e entre o final da estabilização (E10) e ad I ( $Z=-2,215$, p = 0,027); houve queda significativa no desempenho entre a adaptação I e II $(\mathrm{Z}=-2,857 ; \mathrm{p}=0,040)$.

No grupo dos adultos o teste de Friedman também indicou existir diferenças entre os três momentos $\left(\chi^{2}=25,872 ; \mathrm{p}=0,00\right)$; o teste de Wilcoxon indicou que houve aumento significativo no desempenho entre o início da estabilização (E2) e a AdII $(Z=-1,977 ; p=0,048)$. Para esse grupo foram desprezados os resultados referentes ao bloco E10, uma vez que, de acordo com as análises iniciais deste estudo, a maioria dos sujeitos deste grupo terminou a fase de estabilização até o quinto bloco de prática.

O teste de Friedman não indicou diferenças entre as três fases do experimento no grupo de idosos $\left(\chi^{2}\right.$ $=6,907 ; \mathrm{p}=0,075)$.
$\mathrm{Na}$ análise intergrupos, usando o segundo bloco da estabilização (E2) e os últimos blocos da AdI e AdII, o teste de Kruskal Wallis evidenciou diferença significativa entre os grupos somente na AdI $\left(\chi^{2}=8,256 ; p=0,016\right)$ e na AdII $\left(\chi^{2}=14,476 ; p=0,001\right)$. O teste U de Mann Whitney utilizado como post hoc evidenciou diferença significativa na AdI entre adultos e crianças $(Z=-2,599$; $\mathrm{p}=0,009)$ e entre adultos e idosos $(\mathrm{Z}=-2,333 ; \mathrm{p}=$ 0,020 ), sempre com superioridade dos adultos. O mesmo aconteceu na AdII, ocorrendo diferença significativa entre adultos e crianças $(Z=-3,306 ; p=0,001)$ e entre adultos e idosos $(Z=-3,187 ; p=0,001)$. Não houve diferenças entre crianças e idosos na AdI e AdII.

Ao observar a porcentagem de sequências funcionais em cada uma das fases (FIGURA 4), pode se notar que, em termos médios, os adultos na estabilização e nas fases de AdI e AdII mostraram um desempenho evidentemente superior. Devido à falta de respostas dos grupos de crianças e o baixíssimo desempenho dos idosos, não foi possível utilizar teste inferencial para esta variável.

O QUADRO 2 sintetiza os principais resultados intergrupos para todas as variáveis.

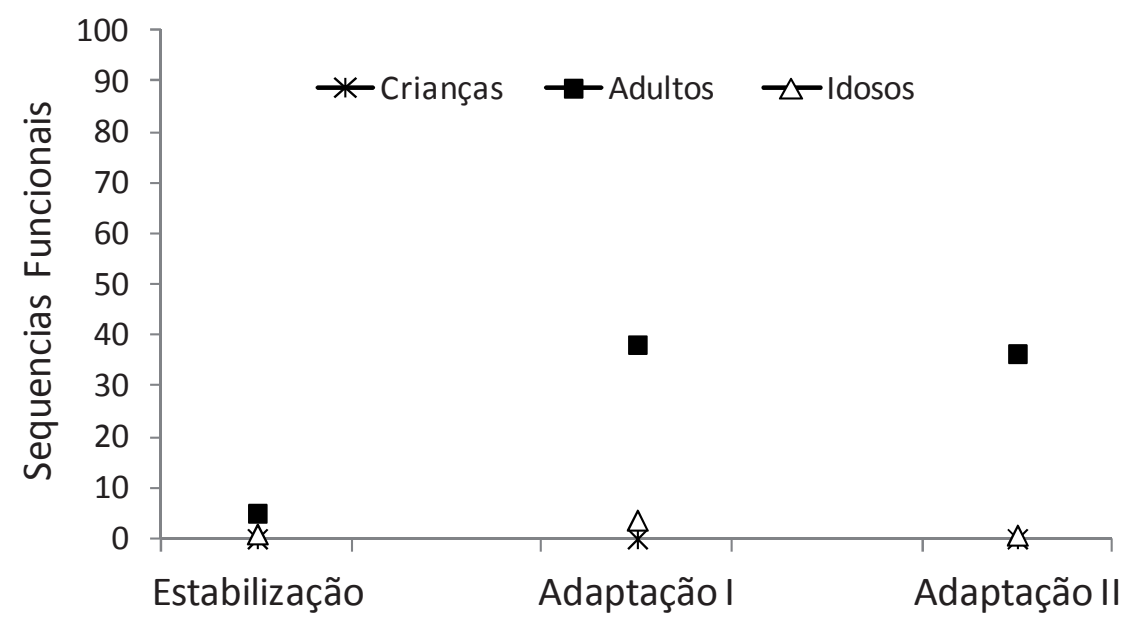

FIGURA 4 - Sequências funcionais (\%) para os grupos de adultos, crianças eidosos, durantea fase deestabilização, adaptação e adaptação II. 
QUADRO 2 - Resumo das diferenças intergrupos nas Fases de Estabilização, Adaptação I e Adaptação II, nas variáveis respostas funcionais, respostas não-funcionais omissas e sequências funcionais.

\begin{tabular}{|lccc|}
\hline Diferenças significativas & Fase de Estabilização & Teste de Adaptação I & Teste de Adaptação II \\
\hline Em respostas funcionais & Idosos $>$ Crianças & Adultos $>$ Crianças e & Adultos $>$ Crianças e \\
Em respostas não-funcionais omissas & Idosos $>$ Crianças & Idosos & Idosos \\
Sequências funcionais & Adultos $>$ Crianças e & Adultos $>$ Crianças e & Adultos > Crianças e \\
& Idosos & Idosos & Idosos \\
\hline
\end{tabular}

O sinal ">" indica "melhor que".

\section{Discussão}

O presente estudo teve por propósito investigar o comportamento de aprendizagem motora como um processo contínuo em sujeitos de diferentes níveis de desenvolvimento, usando, para tanto, a abordagem do Processo Adaptativo. Nesta abordagem, uma modificação introduzida durante a prática pode eliciar uma resposta adaptativa que atenda à demanda da tarefa, além de dar oportunidade para o aprendiz tornar-se mais complexo e competente para responder a novos desafios.

Com relação ao efeito da prática, crianças e adultos melhoram seu desempenho em respostas funcionais durante a estabilização, mas esta não parece ter surtido efeito entre os idosos. Estudos indicam que idosos mantêm a sua capacidade de aprendizado, porém de uma forma mais lenta (Aizenstein, Butters, Clark, Figurski, Andrew Stenger, Nebes, Reynolds III \& Carter, 2006; Nejati, Gerusi Farshi, Ashayeri \& AGHDASI, 2008). A tarefa de rastreamento de um padrão seriado, utilizada no presente estudo tinha uma demanda de tempo exigente o que pode ter proporcionado maior dificuldade para os idosos. Segundo ABReu (2000) o desempenho de idosos em relação aos jovens diminui em tarefas que demandem aumento da reserva funcional, ou seja, quanto mais complexa a tarefa, maior dificuldade de realização.

O baixo desempenho de idosos quando comparado com o de adultos também corrobora os resultados de PinHeIro e CorrêA (2005) que analisaram quatro grupos distintos: crianças, adolescentes, adultos e idosos em uma tarefa de "timing" coincidente; os autores também enfatizaram que, em tarefas complexas, idosos tendem a aumentar o tempo de resposta do movimento em virtude de exigências relativas ao processamento de informação.

Quando Fraser, Li e PenhHUNe (2010) compararam adultos e idosos realizando uma tarefa de "tapping" sequencial simultaneamente com uma tarefa de julgamento semântico (menor demanda de processamento de informação) e depois com uma tarefa aritmética (maior demanda de processamento de informação), observaram que os idosos demonstram menor desempenho mesmo em condições de baixa demanda de processamento informacional e esse prejuízo especificamente apareceu no desempenho de precisão motora. Nesta mesma linha de investigação, ERVEN e JANCZURA (2004) analisaram as interaçōes entre níveis de complexidade da tarefa e variáveis que afetam a cognição como o tempo de exposição aos estímulos, pistas e também diferentes demandas processuais. Eles concluíram que tarefas complexas são mais difíceis para os idosos porque aumentam a quantidade de processamento simultâneo na memória de trabalho.

Mas, um achado que parece importante foi o fato dos idosos serem superiores às crianças no início da prática. Tem sido sugerido que a hierarquia de respostas é capaz de revelar a complexidade de um sistema e seu potencial adaptativo diante de uma perturbação. De fato, em estudos com esta tarefa que foram feitos com crianças (TANI, 1995) e adultos jovens (CATTUZzo, 2007) os resultados têm corroborado essa suposição.

No presente estudo, ao ser questionado se o estágio de desenvolvimento é um elemento que tem efeito no processo de aprendizagem, a hierarquia de respostas poderia auxiliar na explicação dos resultados e, assim, as possíveis diferenças entre crianças e idosos passaram a ser exploradas mediante a análise do desempenho das respostas não funcionais. Houve diferença significativa a favor dos idosos no desempenho em respostas omissas. Assim sendo, poder-se-ia especular se os idosos teriam utilizado uma base de conhecimentos adquirida por experiências vividas com tarefas seriadas ao longo de sua vida e, diferentemente das crianças, evitaram omitir-se. Por outro lado, apesar de crianças supostamente 
terem contato com jogos eletrônicos, que poderiam deixá-las propensas à prática de tarefas motoras seriadas, HaYwoOd e GeTCHell (2010) explicam que fatores centrais de atenção, memória e seleção da resposta são mais lentos em crianças, e isso poderia levar à resposta omissa no início da tarefa. Mas, se os idosos tinham alguma vantagem inicial, eles não foram capazes de mantê-la ao longo do processo de aprendizagem. Crianças superaram suas dificuldades iniciais e mostraram-se competentes na formação e adaptação de uma estrutura mental.

A modificação imposta à tarefa na fase de adaptação I verificou a competência dos sujeitos em suplantar um intervalo de esquecimento, testando a estrutura mental supostamente formada durante a prática. Na adaptação I não se esperava melhora ou piora no desempenho, mas que fosse mantido o nível alcançado na estabilização. A evidente e significativa melhora no desempenho dos adultos durante adaptação II está ligada às peculiaridades desta tarefa. A maioria dos adultos estabilizou mediante alcance do critério de descobrir a sequência de luzes. Tal critério parece funcionar como um parâmetro de ordem, pois, após tê-lo atingido, o sistema passa a funcionar num outro nível, denotando o aumento da complexidade do sistema aprendiz. Segundo Park e Shea (2005), o conhecimento de uma sequência é provavelmente o responsável pelo aumento da velocidade e fluência de resposta.

Crianças e adultos foram capazes de mostrar um desempenho significativamente melhor em respostas funcionais tanto na adaptação I quanto na adaptação II, o que sugere não só a manutenção da estrutura mental formada durante a estabilização, mas também o aumento da complexidade de seu sistema. Para as crianças a diminuição do IIE em $100 \mathrm{~ms}$ parece ter perturbado a estrutura formada na estabilização, ao ponto de levar à queda de seu desempenho. Assim, poder-se-ia especular que crianças e adultos assemelharam-se na aquisição da estrutura mental, mas somente os adultos, que provavelmente estão num outro nível de complexidade, não sofreram com a perturbação pela modificação da velocidade de apresentação dos estímulos na sequência. Esses achados parecem também alinhados à proposição de Guadagnoli e Lee (2004) sobre o "ponto ótimo" para aprendizagem: a partir do mesmo nível de incerteza da tarefa os adultos conseguiram utilizar mais informação que os outros sujeitos.

Para os idosos a situação foi distinta: não houve diferença no seu desempenho de respostas funcionais entre a estabilização e a adaptação I. Estudos sugerem que os idosos possam ter uma taxa de capacidade de processamento de informação mais lenta, necessitando de maior quantidade de prática para estabilizar uma estrutura que se tornasse capaz de fazer frente à perturbação, e que o aprendizado implícito em uma tarefa pode não acontecer (NejATI et al. 2008). Desta forma a demanda de tempo da tarefa pode ter sido insuficiente para a formação de uma estrutura, devido a sua condição desenvolvimental, não permitindo assim, a estabilização de tal estrutura, ou não preservando-a em sua memória de trabalho. Neste sentido, as restrições do estágio de desenvolvimento dos idosos e sua interação com as restrições impostas pela tarefa (prática e demandas percepto-motoras), podem ter comprometido o desempenho dos idosos.

A rigor, os resultados de desempenho em realizar as sequências funcionais completas revelaram que somente os adultos foram capazes de aprender a sequência de estímulos luminosos da tarefa de rastreamento de um padrão seriado. Crianças e idosos não alcançaram o objetivo de aprendizagem da tarefa.

Em suma, pode-se sugerir que crianças e idosos assemelham-se no sentido que as restriçóes provenientes de seu estágio de desenvolvimento combinadas com aquelas provenientes da própria tarefa afetaram sua competência adaptativa. A tarefa de rastreamento seriado utilizada exigia do praticante um alto envolvimento cognitivo (era necessário dominar padrôes de espaço e tempo simultaneamente), isso atribuía uma alta dificuldade nominal à tarefa, pois, independentemente da habilidade do praticante e da condição de prática, tais requerimentos perceptuais e motores a qualificavam como difícil (GuAdagnoli \& Lee, 2004). Essa dificuldade nominal quando combinada com a dificuldade funcional, que segundo GUADAGNOLLI e LEE (2004) é a dificuldade que se refere ao quão desafiante a tarefa é relativa ao nível de cada indivíduo- nesse caso o estágio de desenvolvimento - parece ter extrapolando a capacidade destes aprendizes de reduzirem a incerteza e aprenderem a sequência.

Os resultados do presente estudo e a noção de aprendizagem como um fenômeno contínuo de aumento de complexidade (CATTUZZO \& TANI, 2009; TANI, 2005) permitem estabelecer que, antes de qualificar um sistema como mais ou menos eficiente em seu processamento de informaçōes, dever-se-ia classificá-lo como tendo mais ou menos recursos para lidar com a incerteza e aumentar em complexidade. 


\section{Limitações do estudo}

As decisões metodológicas do presente estudo levaram os experimentadores a finalizar a fase de estabilização com até 10 blocos de prática (com um total de 600 tentativas), para evitar a perda de motivação dos sujeitos, observada no estudo piloto. Mas talvez os idosos precisassem de maior quantidade de prática.

Alem disso o intervalo inter-estímulo da tarefa de rastreamento utilizada no presente estudo, foi de $500 \mathrm{~ms}$. Nesse nível de exigência as crianças caracterizadas pela sua pouca experiência motora e os idosos com processamento e movimentos mais lentos talvez não tenham sido capazes de mostrar o seu potencial adaptativo. Assim,

\section{Conclusão}

De acordo com os resultados obtidos pode-se concluir que os adultos tiveram desempenho em respostas funcionais superior na aprendizagem de tarefa de rastreamento de um padrão seriado em relação às crianças e idosos, tanto na formação da estrutura quanto na sua adaptação à perturbação. Somente adultos foram capazes de aprender a sequência completa do padrão seriado. O grupo de idosos apresentou melhor desempenho os recursos para lidar com a incerteza e aumentar em complexidade podem ser dependentes das restriçóes impostas pelo estágio de desenvolvimento do indivíduo, mas também pelas restrições impostas pela tarefa. A pesquisa em comportamento motor, de maneira geral, deveria estar mais atenta ao que HOPKINS, KALVEBOER e GeUZe (1993) chamam de leis de coexistência no desenvolvimento motor: considerando as relações entre diferentes elementos de um sistema, quando um componente muda, um novo estado de coexistência pode emergir, um no qual o resultado ambiental pode consistir, marcadamente, de diferentes propriedades.

que as crianças no início da prática, o que poderia ser explicado pela experiência motora adquirida ao longo da vida, mas eles não foram capazes de adaptar-se com sucesso. As crianças mostraram competência na formação de sua estrutura mental, e responderam à perturbação com queda no seu desempenho. Enfim conclui-se que, na presente amostra, o estágio de desenvolvimento interagiu com a aprendizagem da tarefa seriada.

\title{
Notas
}

CNPq - Edital Universal - Processo no $476940 / 20087$.

FACEPE - Programa de infra-estrutura para jovens pesquisadores - APQ-0955-4.09/08.

\begin{abstract}
Learning of a motor serial skill in different stages of development

This study investigated the learning of a serial tracking task in different developmental stages. Fifteen children, fourteen adults and thirteen elderly practiced the task of tracking a sequence of six light stimuli for ten blocks of trial or until discover the sequence, during the stabilization phase, and two blocks of trial at the adaptation tests I and II. Performance was measured by the functional and non-functional responses and functional sequences. The results showed that adults were superior to the other participants, and elderly participants performed better than children only in the early practice, suggesting that the development stage interacts with the process of motor learning.
\end{abstract}

UNITERMS: Learning; Psychomotor performance; Tracking task; Serial pattern.

\section{Referências}

ABREU, V.P.S. Memória e envelhecimento. 2000. 125 f. Dissertação (Mestrado) - Faculdade de Educação, Universidade Estadual de Campinas, São Paulo, 2000. 
AIZENSTEIN, H.J.; BUTTERS, M.A.; CLARK, K.A.; FIGURSKI, J.L.; ANDREW STENGER, A.V.;NEBES, R.D.;REYNOLDS III, C.F.;CARTER, C.S. Prefrontal and striatal activation in elderly subjects during concurrent implicit and explicit sequence learning. Neurobiology of Aging, Fayetteville, v.27, n.5, p.741-51, 2006.

BRUCKI, S.M.D; NITRINI, R.; CARAMELLI, P.; BETOLUCCI, P.H.F.; OKAMOTO, I.H. Sugestôes para o uso do mini-exame do estado mental no Brasil. Arquivos de Neuro-Psiquiatria, São Paulo, v.61, n.3B, p.777-81, 2003.

CATTUZZO, M.T. O ciclo instabilidade-estabilidade no processo adaptativo em aprendizagem motora. 2007. 247f. Tese (Doutorado em Biodinâmica do Movimento Humano) - Escola de Educação Física e Esporte, Universidade de São Paulo, São Paulo, 2007. CATTUZZO, M.T.; TANI, G. Leituras em biodinâmica e comportamento motor: conceitos e aplicaçôes. Recife: EDUPE, 2009. CHOSHI, K. Aprendizagem motora como um problema mal definido. Revista Paulista de Educação Física, São Paulo, p.16-23, 2000. Suplemento 3.

ERVEN, T.J.C.G.V.; JANCZURA, G. A memória dos idosos em tarefas complexas. Psicologia: Teoria e Pesquisa, Brasília, v.20, n.1, p.59-68, 2004.

FRASER, S.A.; LI, K.Z.H.; PENHUNE, V.B. Dual-task performance reveals increased involvement of executive control in fine motor sequencing in healthy aging. Journal of Gerontology: Psychological Sciences, Washington, v.65B, n.5, p.526-35, 2010. GUADAGNOLI, M.A.; LEE, T.D. Challenge point: a framework for conceptualizing the effects of various practice conditions in motor learning. Journal of Motor Behavior, Washington, v.36, n.2, p.221-4, 2004.

HAYWOOD, K.M.; GETCHELL, N. Desenvolvimento motor ao longo da vida. 5. ed. Porto Alegre: Artmed, 2010. HOPKINS, B.; KALVEBOER, A.F.; GEUZE, R.H. Epilogue: description versus explanation. In: KALVEBOER, A.F.; HOPKINS, B.; GEUZE, R.H. Motor development in early and later childhood: longitudinal approaches: european network on longitudinal studies on individual development. Cambridge: Cambridge University Press, 1993.

KEMPER, S.; HERMAN, R.E.; LIAN, C.H.T. The costs of doing two things at once for young and older adults: talking while walking, finger tapping, and ignoring speech or noise. Psychology and Aging, Arlington, v.18, p.181-92, 2003.

NEJATI ,V.; GARUSI FARSHI, M.T.; ASHAYERI, H.; AGHDASI, M.T. Dual task interference in implicit sequence learning by young and old adults. International Journal of Geriatric Psychiatry, Chichester, v.23, n.8, p.801-4, 2008. NEWELL, K.M.; KENNEDY, J. Knowledge of results and children's motor learning. Developmental Psychology, Washington, v.14, n.5, p.531-6, 1978.

PALAZZIN, A. Aprendizado motor em crianças e adultos normais: semelhanças e diferenças. 2007. Dissertação (Mestrado) - Instituto de Psicologia, Universidade São Paulo, São Paulo, 2007.

PARK, J.-H.; SHEA, C.H. Sequence learning: response structure and effector transfer. The Quarterly Journal of Experimental Psychology,Hover, v.58A, n.3, p.387-419, 2005.

PINHEIRO, J.P.; CORREAA, U.C. Desempenho em uma tarefa complexa de timing coincidente com desaceleração do estímulo visual em indivíduos de diferentes idades. Revista Brasileira de Educação Física e Esporte, São Paulo, v.19, n.1, p.61-70, 2005. RESTLE, F. Theory of serial patterns learning: structural trees. Psychological Review, Washington, v.77, n.6, p.481-95, 1970. SAMPIERI, R.H.; COLLADO, C.F.; LÚCIO, P.B. Metodologia da pesquisa. 3. ed. São Paulo: McGraw Hill, 2006. TANI, G. Hierarchical organization of an action program and the development of skilled actions. Sheffield: Department of Psychology of University of Sheffield, 1995. [Unpublished Technical Report].

TANI, G.; CORRÊA, U.C.; BENDA, R.N.; MANOEL, E.J. O paradigma sistêmico e o estudo do comportamento motor humano. In: TANI, G. (Ed.). Comportamento motor: aprendizagem e desenvolvimento. Rio de Janeiro: Guanabara Koogan, 2005. THOMAS, J.; NELSON, J.K. Métodos de pesquisa em atividade física. 3. ed. Porto Alegre: Artmed, 2002.

ULRICH, B.D.; REEVE, T.G. Studies in motor behavior: 75 years of research in motor development, learning and control. Research Quartely for Exercise and Sport, Washington, v.76, n.2,p.62-70, 2005.

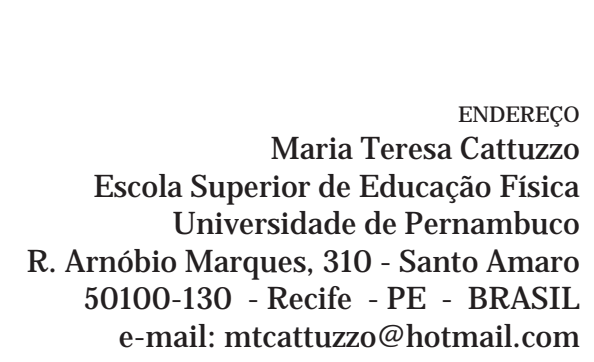

Recebido para publicação: 12/ 05/2011

Revisado: 11/01/2012

Aceito: 13/02/2012 